\title{
Highway Reliability Management Model Based on Depth Recursion for Transportation Demand
}

\author{
Guozhen Sang \\ School of Computer, Weinan Normal University, Weinan 714099, China
}

\begin{abstract}
An effective estimation method for the highway reliability management according to the Zhukov usage model based on the recursive test is put forward. This method makes use of the important sampling technique to ensure that under the conditions of the unbiased reliability estimation, the depth recursion is used to measure the difference between the operation profile and the distribution of the zero variance sampling, to correct the test profile by adjusting the transition probability between all the states through the heuristic iterative process. It has proved theoretically that the reliability of the estimation using the modified test profile test is unbiased estimate with the variance of 0 . Finally, the heuristic iterative algorithm for the generation of the optimal test profile of the highway reliability estimation is given. The simulation results show that the method put forward in this paper can significantly reduce the variance of the estimate compared with the Newton algorithm, and can increase the speed of the recursive test while improving the estimation accuracy at the same time. The research done in this paper can effectively meet the requirements of the transportation industry in the tertiary industry.
\end{abstract}

Keywords: Highway Reliability; Recursive Test; Zhukov Usage Model; Important Sampling; Depth Recursive Method; Transportation industry

\section{Introduction}

Recursive test is a kind of random test method, which carries out the test based on the highway usage model to generate the test instances, makes the recursive analysis according to the test results, and evaluates the quality of highways, which is an important part in the development of highway with high reliability. The recursive test assumes that each data input used has the same defect detection rate. the next operation is selected under the various states according to the probability of transition, and more tests are carried out for the operations that have been executed frequently, which can effectively detect those defects that have relatively great impact on the reliability of the highway. Therefore, the judgment criteria for the adequacy of the recursive test are generally based on the degree of difference between test usage and actual usage, so as to ensure that the reliability estimated based on the test results can represent the reliability of the actual use of the highway [1-2]. This is true for a specific operating environment, which, however, cannot be ensured throughout all the operation sets of the entire highway system. Typical examples are the high-reliability highways, especially the safety critical highways. For the key operations such as the emergency suspension treatment of the nuclear power system, as the probability of its usage is very small, it often cannot be fully tested in the recursive test. However, the reliability index of such operations is often very high, and its failure will often lead to serious consequences. In order to fully test these key operations and obtain the unbiased estimates for the highway reliability, it is necessary to execute a large number of recursive test instances, which will result in the excessively high cost of the recursive testing. On this basis, a number of recursive test methods have been put forward and theoretical research has been carried out [3-4], in which most are the "Control" recursive test methods [5-6]. They mainly make use of the important sampling technique to control the variance of the estimates by correcting the operation profile under the premise of ensuring the estimates of highway reliability or failure risk is unbiased estimation, which has improved the accuracy of the estimates. The key points and difficulties to accurately estimate the reliability of the highway by means of the important sampling method lies in the selection of the sampling distribution, that is, how to determine an optimal test profile, so that the variance estimated after the operation of the test instance generated according to the optimal

ISSN: 0010-8189

(C) CONVERTER 2020

www.converter-magazine.info 
test profile can achieve the minimum.

\section{Measurement of the Degree of Reliability of Zhukov Chain Usage Model}

The Zhukov chain usage model is a Zhukov chain that has the unique initial state and final state, which can be represented using the strongly connected digraph $G=(V, A)$ and the function $p: V \times V \rightarrow[0,1]$. And it has the following properties:

- $V=\{1,2, \ldots n\}$ stands for the set of nodes, which shows the state of use of the highway system;

- A stands for the set of edges, and its elements represent the transition between the states of highway when an operation is selected under a certain state. The edge $e$ from the state $i$ to the state $j$ is defined as an ordered pair $(i, j)$, and there is at most only one directed edge for connection in one direction between any two states, that is, the state $i$ and the state $j$;

- The probability of transition $p(i, j)$ meets $0 \leq p(i, j) \leq 1$, which indicates the probability of the transition from the current state $i$ to the state $j$ in the stage when the program is put into operation

The probability of the operation profile $p$ is defined as the probability that each operation of the highway system is executed in the actual operation, that is, $P=(p(i, j))_{n \times n}$. The test profile $Q$ is the probability that each operation is executed by the highway system during the testing process. Assuming that State 1 is the initial state, State $n$ is the final state, and State $n$ is the state of absorption, which indicates that it will no longer exit once it enters into the State $n$, that is, $p(n, n)=1, p(n, j)=0, \forall j \neq n$. It is further assumed that each state $i \in V$ is accessible from the initial state, that is, there is always a directional path from State 1 to State $i$ existing in $G$. The one-time use of the highway corresponds to a traversal path in the Zhukov usage model from the initial state 1 to the ending state (including the final state $n$ and the failure state), which is denoted as $x=\left(x_{1}, x_{2}, \ldots, x_{L}\right)$, in which, $L$ stands for the total number of operations that are executed in one usage process, that is, the path length. $x_{i}$ is the i-th operation that is traversed in a single usage process, and $x_{L}$ can be the final state $n$ or the failure state. The next operation is executed after the operation $x_{i}$ is executed, which is randomly selected according to the transition probability $p(i, j)$. Therefore, in the course of a single usage of the highway, the probability of the execution of the path $x=\left(x_{1}, x_{2}, \ldots, x_{L}\right)$ is as the following

$$
f(x ; P)=P\{X=x\}=\prod_{i=1}^{L} p\left(x_{i}, x_{j}\right) \text { Satisfy } \sum_{j} p\left(x_{i}, x_{j}\right)=1,
$$

In which, $x$ stands for the execution path that is randomly generated by the operation profile $P, x_{j}$ stands for the state neighboring to the state $x_{i}$ that $x_{j}$ can be transformed into in one step, that is, $p\left(x_{i}, x_{j}\right)>0 . X=\left(X_{1}, X_{2}, \ldots\right)$ stands for the random vector of the execution path. Each test instance of the highway recursive test that is on the basis of the Zhukov chain usage model is corresponding to one of the traversal 
path $x$ as described above. And the indicative function $I_{f}(x)$ is as the following

$$
I_{f}(x)=\left\{\begin{array}{l}
1, \text { In case of failure during the path execution process } \\
0, \text { Otherwise }
\end{array}\right.
$$

Apparently, whether the system is operating properly is relying on the specifications of the highway system. And for a given highway system $\pi$, its specifications are also given. The reliability $R$ of the highway is defined as the probability of failure of the highway during the process of one single usage. It can be known from the definition of $I_{f}(x)$ that the reliability $R(\pi)$ of the system $\pi$ is as the following

$R(\pi)=1-E_{P}\left[I_{f}(X)\right] \sqcap 1-\ell$

In which, $E_{P}$ stands for the solving of the expectation relative to the distribution of the probability $f(x ; P)$, and $\ell$ stands for the failure probability of the system, that is, $\ell=E_{P}\left[I_{f}(X)\right]$.

It is noted that when the test is conducted along a path that contains a circle, there is at least one operation (denoted as $\mathrm{C}$ ) that occurs in the test for multiple times. Therefore, with the increase in the number of times of the execution of operation $\mathrm{C}$, the probability of failure of this path will be approaching to 1 . If there is a circle in the vast majority of the paths that have been executed, it will lead to the excessively low estimation of the reliability of the entire highway system when the number of repetitions in the execution of the operation is large enough. In order to avoid the excessively pessimistic estimation of the reliability, it is assumed that when an operation occurs repeatedly, it shall be considered to have occurred only once [7-8].

\section{Sampling Method for the Reliability of the Highway Based on the Depth Recursion}

\subsection{Import Sampling Method based on the Deep Recursion}

It can be known from the equation (1) that, the most straightforward method to obtain the unbiased estimation of the system reliability is as the following: Select $\mathrm{N}$ traversal paths $x_{1}, x_{2}, \ldots, x_{N}$ according to the Zhukov chain model and obtain directly the unbiased estimation of the probability of failure by the moment estimation as the following:

$\hat{\ell}=\frac{1}{N} \sum_{i=1}^{N} I_{f}(x)$

Thus the unbiased estimate $\hat{R}(\pi)$ of the system reliability can be obtained.

However, for the safety critical highway systems, highway failure is not easy to be observed. To obtain relatively accurate reliability estimates, it is necessary that the number of paths (the number of sampling) $\mathrm{N}$ to be tested is large enough, which will inevitably lead to the excessive high overhead of the test. In order to overcome the defects of this method, the important sampling method is generally used to conduct the testing by selecting the appropriate sampling profile, that is, the test profile $Q$, increasing the probabilities of the traverse for the rare state, and improving the opportunity to observe the failure of the highway. To ensure that the estimation carried out

ISSN: 0010-8189

(C) CONVERTER 2020

www.converter-magazine.info 
according to the test profile is unbiased estimation, the likelihood ratio is often used to carry out the weighted adjustment, because

$$
\ell=E_{P}\left[I_{f}(X)\right]=\int I_{f}(x) \frac{f(x ; P)}{f(x ; Q)} f(x ; Q) d x=\int I_{f}(x) W(x, P, Q) f(x ; Q) d x(3)
$$

In which, $W(x, P, Q)=f(x ; P) / f(x ; Q)$ stands for the likelihood ratio. Therefore, the unbiased estimate of the failure probability $\ell$ can be denoted as the following

$$
\hat{\ell}=\frac{1}{N} \sum_{i=1}^{N} I_{f}\left(x_{i}\right) W\left(x_{i}, P, Q\right)
$$

In which, $x_{i}$ stands for the test instance that is randomly generated according to the test profile $Q$

It is noted that in the equation (4), when the optimal test profile $Q^{*}$ meets the form of the following equation (5), the unbiased estimate of the failure probability with the variance of 0 can be obtained just by randomly generating a test path according to the optimal test profile $Q^{*}$, that is, the test profile that meets the equation (5) is the sampling distribution with the variance of zero.

$$
f\left(x ; Q^{*}\right)=\frac{I_{f}(x) \cdot f(x ; P)}{\ell}
$$

However, the optimal test profile $Q^{*}$ that meets the equation (5) is a function of the true value $\ell$, which is not known in the practical application. Therefore, it is not realistic to conduct the sampling using the $Q^{*}$. It can be known from the form of $f\left(x ; Q^{*}\right)$ that the probability distribution $\{f(\cdot ; Q)\}$ that is closest to $f\left(x ; Q^{*}\right)$ in the probability distribution cluster $\{f(\cdot ; Q)\}$ of $f(\cdot ; P)$ can be identified. Thus the parameter $Q$, that is, the optimal (or asymptotically optimal) test profile, can be determined, so that the unbiased estimate with the variance of zero or close to 0 can be obtained. The K-L distance or depth recursion is commonly used to describe the difference between two probability distributions.

In this way, the problem of determining the optimal test profile is transformed

Hence, the problem to determine the optimal test profile can be transformed into the determination of the inference parameter $Q$, so that the depth recursion of $f(x ; Q)$ with the probability distribution $f\left(x ; Q^{*}\right)$ is minimized, that is, the following can be obtained

$$
Q^{*}=\arg \min _{Q}\left\{-\int f\left(x ; Q^{*}\right) \ln f(x ; Q) d x\right\}
$$

Introduce the equation (5) to the equation (6), the following equivalent inference parameter determination form can be obtained: 


$$
Q^{*}=\arg \max \left\{\int I_{f}(x) f(x ; P) \ln f(x ; Q) d x\right\}=\arg \max _{Q}\left\{E_{P}\left[I_{f}(x) \ln f(x ; Q)\right]\right\}
$$

It is noted that $f(x ; Q)=\prod_{i=1}^{L} q\left(x_{i}, x_{j}\right)$ meets $\sum_{j} q\left(x_{i}, x_{j}\right)=1$. Then, it can be known from the method of Lagrange multipliers, the equation (7) can be transformed into the following optimization problem for solution:

$$
\max _{Q}\left\{E_{P}\left[I_{f}(x) \sum_{i} \ln q\left(X_{i}, X_{i+1}\right) \ln f(X ; Q)\right]\right\}+\sum_{i} u_{i}\left(\sum_{j} q\left(X_{i}, X_{j}\right)-1\right)(8)
$$

In which, $u_{i}$ stands for the Lagrangian factor. Seek the partial derivative for the above equation on $q\left(x_{l}, x_{m}\right)$, let it equal to 0 , and the element $q\left(x_{l}, x_{m}\right)$ of $Q^{*}$ can be obtained as the following

$$
q\left(x_{l}, x_{m}\right)=E_{P}\left[I_{f}(X) \sum_{i} I_{\left\{X_{i}=x_{l}, X_{i+1}=x_{m}\right\}}\right] / E_{P}\left[I_{f}(X) \sum_{i} I_{\left\{X_{i}=x_{l}\right\}}\right]
$$

Therefore, the optimal test profile for the estimation of the highway reliability based on the important sampling method can be obtained.

\subsection{Nature of the Highway Reliability Estimation}

Property 3.1. If $f(x ; P)>0$, the elements of the test profile $Q$ meet the equation (9), then the reliability estimated by the equation (4) and equation (1) are unbiased estimates.

Prove: As $\int I_{f}(x) W(x, P, Q) f(x ; Q) d x=\int I_{f}(x) \frac{f(x ; P)}{f(x ; Q)} f(x ; Q) d x=E_{P}\left[I_{f}(X)\right]=\ell$, therefore, it can be known that, the equation (4) is the unbiased estimation of $\ell$. From $R(\pi)=1-E_{P}\left[I_{f}(X)\right]=1-\ell$, the unbiased estimation of the reliability $R(\pi)$ can be obtained, quod erat demonstrandum.

In order to obtain the property of the variance of the estimates of the test profile sampling according to the equation (9) that the elements meet, it is necessary to make use of the following property of the important sampling method: Lemma 3.2. Let the random variable (vector) have the probability density function $f(x)>0$, then the important sampling estimate of $x \in R^{1}$ integral $I=\int h(x) d x$ is as the following

$\hat{g}(X)=\frac{1}{N} \sum_{i=1}^{N} \frac{h\left(X_{i}\right)}{f\left(X_{i}\right)}, X_{i} \square f(x)$

If for $\forall x \in\{x: f(x)>0\}$, there is the existence of constant $c$ that meets $h(x)=c f(x)$, the important sample estimate $\hat{g}(X)$ is the unbiased estimate of the integral $I$ with the variance of 0 . 
Prove: It is clear that $\hat{g}(X)$ is the unbiased estimate of the integral $I$. It is denoted that $s(x)=h(x) / f(x)$, then the following can be obtained

$\hat{g}(X)=\frac{1}{N} \sum_{i=1}^{N} s\left(X_{i}\right)$, thus the variance of $\hat{g}(X)$ can be obtained as the following

$\operatorname{Var}(\hat{g}(X))=\operatorname{Var}\left(\frac{1}{N} \sum_{i=1}^{N} s\left(X_{i}\right)\right)=\frac{\operatorname{Var}\left(s\left(X_{i}\right)\right)}{N}=\frac{1}{N} \int[s(x)-E(s(X))]^{2} f(x) d x$

For $\forall x \in\{x: f(x)>0\}$, there is the existence of constant $c$ that meets $h(x)=c f(x)$. Therefore, it is obvious that $h(x) / f(x)=c$, and $E[s(X)]=E[h(X) / f(X)]=c$ is established. Hence, the variance of $\hat{g}(X)$ can be obtained as 0 , quod erat demonstrandum.

Similar to the literature [9], the following can be obtained using Lemma 3.2:

Property 3.3. If $f(X ; P)>0$, the elements of the test profile $Q$ meet the equation (9), then the reliability estimated by the equation (4) and the equation (1) is the unbiased estimate with the variance of zero.

Prove: The estimated unbiasedness is guaranteed by the property 3.1, and the variance of the estimation is proved to be 0 as the following. It is easy to known that, to prove that the variance of the reliability estimates is 0 , it is only required to prove that the estimates of the failure probability have zero variance. It can be known from Lemma 3.2 that, if the likelihood ratio function $W(x, P, Q)$ of the failure probability estimates is constant, it can be proved that the variance of the estimates is 0 . Assuming that $x=\left(x_{1}, x_{2}, \ldots, x_{L}, x_{L+1}\right)$ is the failure test paths finally observed in one test execution process, then the probability of the execution of the selection of path $x$ according to the operation profile $P$ is $f(x ; P)=\prod_{i=1}^{L} p\left(x_{i}, x_{i+1}\right)$. And the probability of the execution of the selection of path $x$ according to the test profile $Q$ is $f(x ; Q)=\prod_{i=1}^{L} q\left(x_{i}, x_{i+1}\right)$. From the properties of the indicative function $I_{f}(x)$ and the Zhukov property of the state transition, the numerator of the equation (9) can be expressed as the following,

$$
\begin{aligned}
E_{P}\left[I_{f}(X) \sum_{i=1}^{L} I_{\left\{X_{i}=x_{l}, X_{i+1}=x_{m}\right\}}\right] & =\sum_{i=1}^{L} E\left\{I_{f}(X)=1, X_{i}=x_{l}, X_{i+1}=x_{m}\right\} \\
& =\sum_{i=1}^{L} P\left\{I_{f}(X)=1 \mid X_{i+1}=x_{m}\right\} P\left\{X_{i+1}=x_{m} \mid X_{i}=x_{l}\right\} P\left\{X_{i}=x_{l}\right\}^{(11)} \\
& =\sum_{i=1}^{L} r_{m} P\left(x_{l}, x_{m}\right) P\left\{X_{i}=x_{l}\right\}
\end{aligned}
$$

In which, $r_{m}$ stands for the probability that is observed to have occurred in the system starting from the state $m$ to the state before it enters the final state. Similarly, the denominator of the equation (9) can be denoted as the following

ISSN: 0010-8189

(C) CONVERTER 2020 
$E_{P}\left[I_{f}(X) \sum_{i=1}^{L} I_{\left\{X_{i}=x_{l}\right\}}\right]=\sum_{i=1}^{L} r_{l} \cdot P\left\{X_{i}=x_{l}\right\}$

Introduce the equation (11) and equation (12) to the equation (9), and the transition probability can be obtained as the following:

$q\left(x_{l}, x_{m}\right)=\frac{\sum_{i=1}^{L} r_{m} P\left(x_{l}, x_{m}\right) P\left\{X_{i}, x_{l}\right\}}{\sum_{i=1}^{L} r_{l} \cdot P\left\{X_{i}=x_{l}\right\}}=\frac{r_{m} P\left(x_{l}, x_{m}\right)}{r_{l}}$

Hence the following can be obtained:

$f(x ; Q)=\prod_{i=1}^{L} q\left(x_{i}, x_{i+1}\right)=\frac{r_{x_{2}} p\left(x_{1}, x_{2}\right)}{r_{x_{1}}} \cdot \frac{r_{x_{3}} p\left(x_{2}, x_{3}\right)}{r_{x_{2}}} \cdot \ldots \cdot \frac{r_{x_{L+1}} p\left(x_{L}, x_{L+1}\right)}{r_{L}}$

Thus, the likelihood ratio $W(x, P, Q)$ can be denoted as the following

$W(x, P, Q)=\frac{f(x ; P)}{f(x ; Q)}=\frac{r_{x_{1}}}{r_{L+1}}$

It is noted that $x_{1}$ is the initial state, and $x_{L+1}$ is the failure state. Since $x$ is the test path that is finally observed in the test execution process, there must be $r_{L+1}=1$. Therefore, the likelihood ratio of the test path $x$ is $r_{1}$. It can be known from the definition of $r_{1}, r_{1}$ is the constant that is independent of the test path. And since all the samples in the equation (9) are all the test paths of failure $I_{f}(x)$ finally observed, each test path has the same constant likelihood ratio $r_{1}$. It can be known from Lemma 3.2 that, the variance estimated by $\ell$ is 0 . It can be further obtained that the variance estimated for the reliability $R(\pi)$ is also 0 , quod erat demonstrandum.

\subsection{Generation of the Optimal Test Profile}

It is noted that the sampling distribution of the optimal test profile Q generated by the equation (9) is the operating profile $\mathrm{P}$, which is the key operation for the safety critical highway. As the probability in the actual use is relatively small, it will be very difficult to observe the failure when sampling is carried out according to $P$. Again, the important sampling method is used to generate a test profile sequence $\left\{Q_{0}^{*}, Q_{1}^{*}, \ldots\right\}$ by a kind of recursive algorithm. The next iteration of test path is generated using the approximate test profile obtained from the previous iteration to obtain a new test profile until the test profile sequence converges.

In fact, $Q_{0}^{*}$ is initialized to the operating profile $\mathrm{P}$ in the first step of the iteration, that is, $Q_{0}^{*}=P$. Through the stochastic simulation, the first near-optimal solution $Q_{1}^{*}$ of the equation (9) can be obtained as the following:

$Q_{1}^{*}=\arg \max _{Q}\left\{E_{P}\left[I_{f}(x) \ln f(X ; Q)\right]\right\} \approx \arg \max _{Q} \frac{1}{N} \sum_{i=1}^{N} I_{f}\left(x_{i}\right) \ln f\left(x_{i} ; Q\right)$

ISSN: 0010-8189

(C) CONVERTER 2020 
In the second iteration process, $N$ test paths are sampled according to $Q_{1}^{*}$ to generate the second near-optimal solution $Q_{2}^{*}$ as the following:

$$
\begin{aligned}
Q_{2}^{*} & =\arg \max _{Q}\left\{E_{P}\left[I_{f}(X) \ln f(X ; Q)\right]\right\} \\
& =\arg \max _{Q}\left\{\int I_{f}\left(x_{i}\right) f(x ; P) \frac{f\left(x ; Q_{1}^{*}\right)}{f\left(x ; Q_{2}^{*}\right)} \ln f(x ; Q) d x\right\} \\
& =\arg \max _{Q} E_{Q_{1}^{*}}\left[I_{f}\left(x_{i}\right) W\left(x, P, Q_{1}^{*}\right) \ln f(X ; Q)\right] \\
& \approx \arg \max _{Q} \frac{1}{N} \sum_{i=1}^{N} I_{f}\left(x_{i}\right) W\left(x, P, Q_{1}^{*}\right) \ln f\left(x_{i} ; Q\right)
\end{aligned}
$$

Thus the calculation equation for the asymptotical optimal test profile $Q_{j}^{*}$ in the $j$-th iteration can be obtained as the following

$$
\begin{aligned}
Q_{j}^{*} & =\arg \max _{Q}\left\{E_{P}\left[I_{f}(X) \ln f(X ; Q)\right]\right\} \\
& \approx \arg \max _{Q} \frac{1}{N} \sum_{i=1}^{N} I_{f}\left(x_{i}\right) W\left(x_{i}, P, Q_{j-1}^{*}\right) \ln f\left(x_{i} ; Q\right)
\end{aligned}
$$

It is noted that $f(x ; Q)=\prod_{i=1}^{L} q\left(x_{i}, x_{j}\right)$ meets $\sum_{j} q\left(x_{i}, x_{j}\right)=1$, then the solution of the above equation can be solved by transforming into the problem of maximum value according to the method of Lagrange multipliers:

$$
\max _{Q}\left\{E_{Q_{j-1}^{*}}\left[I_{f}(X) W\left(x_{i}, P, Q_{j-1}^{*}\right) \sum_{j} \ln q\left(x_{i}, x_{j}\right)\right]\right\}+\sum_{i} u_{i} \sum_{j} q\left(X_{i}, X_{j}\right)
$$

Seek the partial conductance for the above equation relative to $q\left(x_{l}, x_{m}\right)$, let it equal to 0 , and the element $q\left(x_{l}, x_{m}\right)$ of $Q_{j}^{*}$ can be obtained as the following

$$
q\left(x_{l}, x_{m}\right)=\frac{E_{Q_{j-1}}\left[I_{f}(X) W\left(X, P, Q_{j-1}\right) \sum_{i} I_{\left\{X_{i}=x_{l}, X_{i+1}=x_{m}\right\}}\right]}{E_{Q_{j-1}}\left[I_{f}(X) W\left(X, P, Q_{j-1}\right) \sum_{j} I_{\left\{X_{i}=x_{l}\right\}}\right]}
$$

Thus the estimate equation ^ (,) of $q\left(x_{l}, x_{m}\right)$ can be obtained as the following 
$\hat{q}\left(x_{l}, x_{m}\right)=\frac{\sum_{k=1}^{N} I_{f}\left(x_{k}\right) W\left(x_{k}, P, Q_{j-1}\right) \sum_{i} I_{\left\{X_{i}=x_{l}, X_{i+1}=x_{m}\right\}}}{\sum_{k=1}^{N} I_{f}\left(x_{k}\right) W\left(x_{k}, P, Q_{j-1}\right) \sum_{i} I_{\left\{X_{i}=x_{l}\right\}}}$

In which, the test path $x_{k}, \mathrm{k}=1,2, \ldots, N$ is randomly generated by the test profile $Q_{j-1}$. When the indicative function $I_{\left\{X_{i}=x_{l}\right\}}$ takes the value of 1 , it indicates that the test path $x_{k}$ has executed the operation $x_{l}$; otherwise, it takes the value of 0 . When $I_{\left\{X_{i}=x_{l}, X_{i+1}=x_{m}\right\}}$ takes the value of 1, it indicates that after the test path $x_{k}$ completes the execution of the operation $x_{l}$, it continues to execute the operation $x_{m}$; otherwise, it takes the value of 0 .

In this paper, an iterative algorithm based on the depth recursion can be used to solve the optimal or asymptotic optimal test profile, and then the important sampling method is adopted through the optimal test profile to estimate the reliability of the highway system: The initialized test profile $Q_{0}$ is the test path randomly generated by the operation profile $P$. The equation (18) is used to correct the test profile $Q_{1}$ and then the corrected test profile $Q_{1}$ can be further used for the sampling, and the cycle is repeated, ..., until the test profile no longer has any changes or the change is not very great. The specific algorithm is as the following:

Algorithm 4.1. Depth recursive algorithm for the estimation of the highway reliability

1. Initialize the test profile $\hat{Q}_{0}$ into the operating profile $P$, and the number of iterations $j=1$.

2. Generate the test paths $x_{1}, x_{2}, \ldots, x_{N}$ according to the test profile $\hat{Q}_{j-1}$ and observe whether failure of highway occurs.

3. From the generated $\mathrm{N}$ test paths $x_{1}, x_{2}, \ldots, x_{N}, \tilde{Q}_{j}$ is solved by the equation (18), and the smoothing technique is applied to obtain the following:

$$
\hat{Q}_{j}=\alpha \tilde{Q}_{j}+(1-\alpha) \hat{Q}_{j-1}, \alpha \in(0.4,0.9)
$$

4. Let $j=j+1$, repeat step 2 and step 3 until $\hat{Q}_{j}=\hat{Q}_{j-1}=\ldots=\hat{Q}_{j-d}$ is established for a certain given $d$ (in case $d=4$ ) or the maximum change in two adjacent test profiles is less than the given $\varepsilon$.

5. According to the generated test profile $\hat{Q}_{j}$, randomly generate $\mathrm{M}$ test paths $x_{1}, x_{2}, \ldots, x_{m}$, and the reliability of the highway can be estimated using the following equation:

$\hat{R}(\pi)=1-\frac{1}{M} \sum_{i=1}^{M} \frac{f\left(x_{i} ; P\right)}{f\left(x_{i} ; \hat{Q}_{j}\right)} \cdot I_{f}\left(x_{i}\right)$.

In the step 3, when the test profile is corrected by using the equation (18), the probability of transition from the current state to the neighboring state remains to be the corresponding transition probability in the previous iteration unchanged if the denominator is 0 . In addition, in the step 3 , the smoothing techniques are used mainly to reduce certain elements of $\hat{Q}_{j}$ as 0 , so as to prevent the algorithm from keeping on searching for the local optimal solution from the initial stage. 


\section{Example Analysis}

In order to illustrate the highway reliability management method based on the recursive testing, highway vehicle instance is used for the verification, as shown in Figure 1. The highway consists of 12 operations. The execution each time starts from Operation 1, and Operation 12 is the operation executed at the end of the operation of the highway. Edge $(4,6),(8,9)$ and $(8,10)$ trigger the execution of the key operation 6,9 , and 10 . The priori probabilities of the respective operational failure are represented by the vector $f$ as the following:

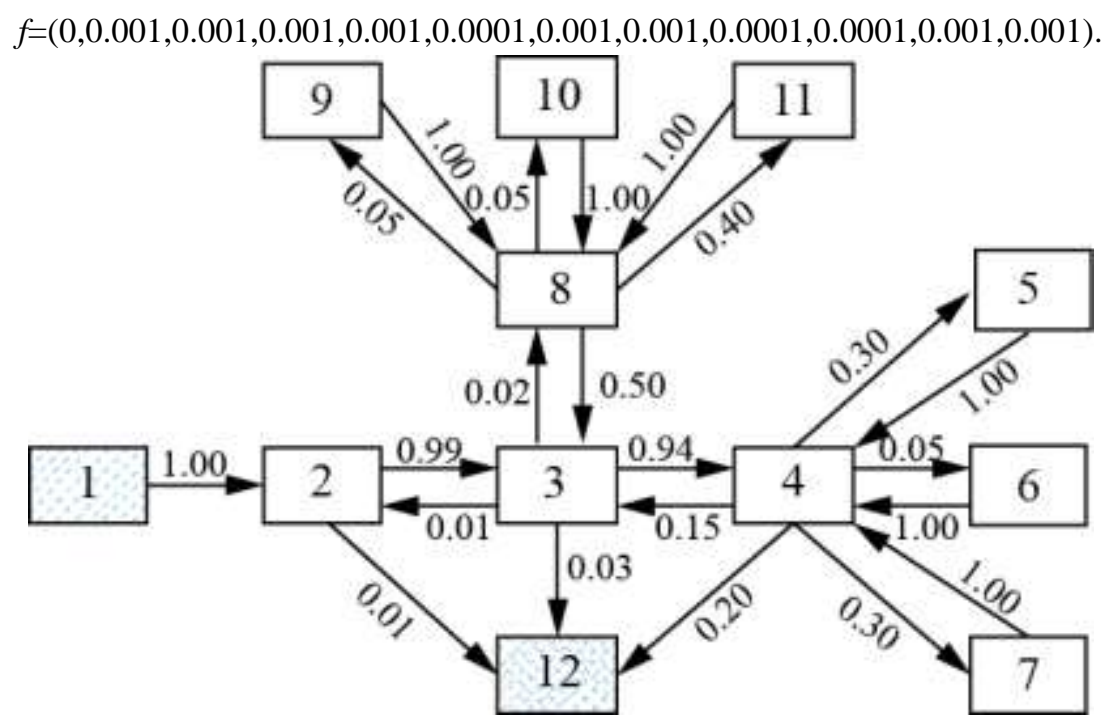

Figure 1. Zhukov chain usage model put forward by Gutjahr

The Newton algorithm [10] and the depth recursion (CE) method as well as the standard recursive test method (Standard) (using the operating profile $\mathrm{P}$ ) are adopted to conduct sampling of $\mathrm{N}=2000$ test paths and carry out the simulation test for 100 times. And 100 estimated values of the reliability of the highway system are obtained. The reliability estimation and variance under in three cases are shown in Fig. 2 and Fig. 3, respectively.

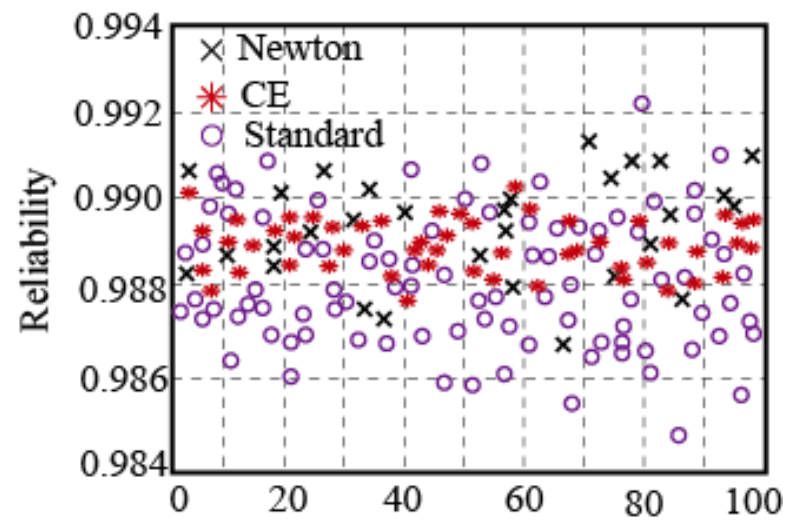

Figure 2. Estimates of the reliability under the 3 different profiles

ISSN: 0010-8189 


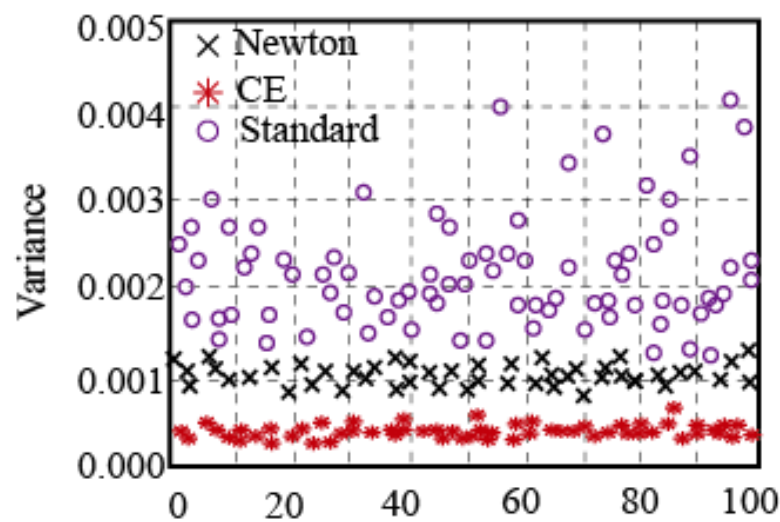

Figure 3. Variance of the reliability estimates under the 3 different profiles

In the 100 simulations, the mean value of the reliability estimated by the CE method is 0.9891 and the variance is $4.36 \times 10^{-4}$. The mean value of the reliability estimated by the SA algorithm is 0.9893 and the variance is $1.14 \times$ $10^{-3}$. The mean value of the reliability estimated by the recursive test method is 0.9889 and the mean variance is $2.46 \times 10^{-3}$. It can be seen that the recursive method can significantly reduce the estimated variance compared with that of the Newton algorithm and the standard method. The box plots in the three cases are shown in Figure 4.

It can be seen from the box plot that the reliability estimated by the depth recursive method is relatively concentrated in the vicinity of the median, and the dispersion degree obtained by the Newton algorithm is relatively larger, while the dispersion degree of the estimated value obtained by the standard method is the greatest. In 100 simulations, the traversal paths each time are 2,000 paths. When the standard method is used for simulation, the cumulative number of times that the operation 6 is traversed is 20,576, and the cumulative numbers of times that the key operation 9 and operation 10 are traversed are 476 times and 514 times, respectively. In the case of the adoption of the depth recursive method, the number of times that the operation 6 is traversed is 79,394, and the cumulative numbers of times that the key operation 9 and operation 10 are traversed are 4,550 times and 4,468 times, respectively. When the Newton algorithm is adopted, the cumulative number of times that the operation 6 is traversed is 55,090, and the cumulative numbers of times that the operations 9 and operation 10 are traversed are 10,898 and 8,162, respectively. Therefore, it can be seen that the adoption of both the depth recursive method and the Newton algorithm can significantly improve the traversal opportunity of the rare operation. And the deep recursive method has greater opportunity of traversing the critical operation 6 than that of the Newton algorithm, while its opportunity of traversing the critical operation 8 and operation 9 is lower than that of the Newton algorithm.

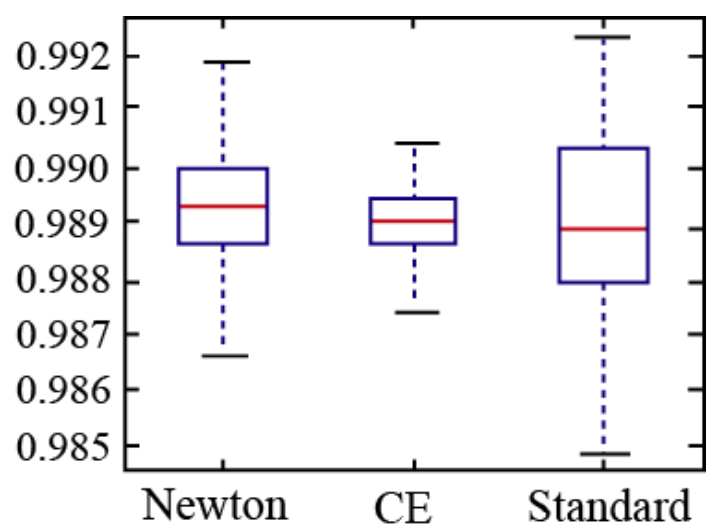

Figure 4. Box plot for the estimates of the reliability under the 3 different profiles

\section{CONCLUSION}

In this paper, based on the important sampling technique, a kind of correction mechanism is used to adjust the

ISSN: 0010-8189

(C) CONVERTER 2020 
operation profile by using the recursive method. The variance of the estimates is reduced under the premise that the reliability estimation being the unbiased estimation is guaranteed, giving full play to the advantages of the important sampling technique, increasing the test opportunities to use the key operations with small probability of use and speeding up the highway test. In this method, the correction of the test profile is transformed into an optimization problem to seek the iterative solution, estimate the reliability of the highway and speed up the highway test. The defect of solving the optimal test profile by using the depth recursive method is that: The simulation cost required is greater than that of the Newton algorithm and the standard method, and it is impossible to obtain the precise solution of the optimal test profile, but can only obtain a near-optimal solution that meets the accuracy of the reliability estimation.

\section{References}

[1] Fenton G A, Naghibi F, Dundas D, et al., "Reliability-based geotechnical design in 2014 Canadian Highway Bridge," Journal of Geotechnical Engineering, vol. 53, no. 2,pp.236-251,2015.

[2] Zhu J, Chen C, Han Q, "Vehicle-bridge coupling vibration analysis based fatigue reliability prediction of prestressed concrete highway bridges," Structural Engineering \& Mechanics, vol.49, no. 2, pp.203-223, 2014.

[3] Ghosh J, Rokneddin K, Padgett J E, et al., "Seismic Reliability Assessment of Aging Highway Bridge Networks with Field Instrumentation Data and Correlated Failures, I: Methodology,'Earthquake Spectra, no. 30, pp.795-817, 2014.

[4] Wakabayashi H, Matsumoto Y, "Comparative study on travel time reliability indexes for highway users and operators," Journal of Advanced Transportation, vol. 46, no. 4, pp.318-339, 2012.

[5] Ghosh J, Caprani C C, Padgett J E, "Influence of Traffic Loading on the Seismic Reliability Assessment of Highway Bridge Structures,’Journal of Bridge Engineering, vol. 19, no. 3, 2014.

[6] Catelani M, Ciani L, Paolilli E S, "Reliability and availability analysis of an automatic highway toll collection system,'Instrumentation and Measurement Technology, pp.1594-1598, 2013.

[7] Wang H, Sun H, Qin K, "Estimating Recursion Depth for Loop Subdivision," International Journal of Cad/cam, vol. 4, no. 1, pp.94-98, 2014.

[8] Siochi R A, "Optimized removal of the tongue-and-groove underdose via constrained partial synchronization and variable depth recursion,” Physics in Medicine \& Biology, vol.54, no. 5, pp.1369, 2016.

[9] Hrubcová P, Środa P, Vavryčuk V, et al., "Comment on the Seismic Method Depth-Recursive Tomography on Grid (DRTG) Developed by Miroslav Novotný and Recently Published in Three Papers in Surveys in Geophysics, " Surveys in Geophysics, vol.34, no. 4,pp.521-529,2013.

[10] Cornelius B J, Kirby G H, "Depth of recursion and the ackermann function," Bit Numerical Mathematics, vol.15, no.2,pp.144-150, 2013. 